\title{
TOPOLOGIES BETWEEN COMPACT AND UNIFORM CONVERGENCE ON FUNCTION SPACES
}

\author{
S. KUNDU \\ Division of Theoretical Statistics and Mathematics \\ Indian Statistical Institute \\ 203 B.T. Road, Calcutta 700035 \\ India \\ and \\ R.A. McCor \\ Department of Mathematics \\ Virginia Polytechnic Institute \& State University \\ 460 McBryde Hall \\ Blacksburg, VA 24061-0123 \\ U.S.A.
}

(Received June 27, 1991 and revised form July 31, 1991)

\begin{abstract}
This paper studies two topologies on the set of all continuous real-valued functions on a Tychonoff space which lie between the topologies of compact convergence and uniform convergence.
\end{abstract}

KEY WORDS AND PHRASES. Function spaces, Compact-open topology, Topology of uniform convergence, $\sigma$-compact, Pseudocompact.

1991 AMS SUBJECT CLASSIFICATION CODES. 54C35, 54D45.

\section{INTRODUCTION.}

The set $C(X)$ of all continuous real-valued functions on a Tychonoff space $X$ has a number of natural topologies. Two commonly used topologies are the compact-open topology and the topology of uniform convergence. The latter topology has been used for more than a century as the proper setting to study uniform convergence of sequences of functions. The compact-open topology made its appearance in 1945 in a paper by Fox [3], and soon after was developed by Arens in [1] and Arens and Dugundji in [2]. This topology was shown in [6] to be the proper setting to study sequences of functions which converge uniformly on compact subsets. One of the distinguishing features of this topology is that whenever $X$ is locally compact the compact-open topology on $C(X)$ is the coarsest topology making the evaluation map $e: X \times C(X) \rightarrow \Re$ continuous (where $e(x, f)=f(x)$ ).

The compact-open topology and the topology of uniform convergence are equal if and only if $X$ is compact. Because compactness is such a strong condition, there is a considerable gap between these two topologies. This gap was especially felt in [8] while studying the completeness of a normed linear space of continuous linear functionals on $C(X)$ with the compact-open topology. Because of this, a new class of topologies was introduced in [7] on $C^{*}(X)$ to bridge the gap, where $C^{*}(X)$ is the set of bounded functions in $C(X)$. This also generalized the $\sigma$-compact-open topology 
on $C^{*}(X)$ which was studied in [5]. The purpose of this present work is to extend these ideas to two natural topologies on $C(X)$, to study the properties of these topologies, and to relate these topologies to the compact-open topology and the topology of uniform convergence.

Let $C_{k}(X)$ and $C_{u}(X)$ denote $C(X)$ with the compact-open topology and the topology of uniform convergence, respectively. The definitions of the other two topologies that we study here are based on the fact that $C_{k}(X)$ can be viewed in two different ways. First we can view the compact-open topology as a "set-open topology," where a subbasic open set looks like

$$
[K, V]=\{f \in C(X): f(K) \subseteq V\},
$$

where $K$ is a compact subset of $X$ and $V$ is an open subset of the reals. Note that $V$ can always be taken as a bounded open interval. The second way that we can view the compact-open topology is as a "uniform topology." This approach is developed in the next section.

Throughout the rest of the paper, we use the following conventions. All spaces are Tychonoff spaces. If $X$ and $Y$ are any two spaces with the same underlying set, then we use $X=Y, X \leq Y$ and $X<Y$ to indicate, respectively, that $X$ and $Y$ have the same topology, that the topology on $Y$ is finer than or equal to the topology on $X$, and that the topology on $Y$ is strictly finer than the topology on $X$. The symbols $\Re$ and $N$ denote the spaces of real number and natural numbers, respectively. Finally, the constant zero-function in $C(X)$ is denoted by $f_{0}$.

1. TOPOLOGIES OF UNIFORM CONVERGENCE.

In this first section we look at "uniform topologies" on $C(X)$ in a general setting. This is done in terms of certain pseudo-seminorms on $C(X)$. By a pseudo-seminorm on a real linear space $E$ is meant a real-valued function $p$ on $E$ such that

(1) $p(0)=0$,

(2) $p(x)=p(-x)$ for all $x \in E$, and

(3) $p(x+y) \leq p(x)+p(y)$ for all $x, y \in E$.

Note that it immediately follows that $p(x) \geq 0$ and $|p(x)-p(y)| \leq p(x-y)$ for all $x, y \in E$.

A pseudo-seminorm $p$ is called a seminorm if the following additional condition holds.

(4) $p(t x)=|t| p(x)$ for all $x \in E$ and $t \in \Re$.

Of course a seminorm $p$ is a norm if $p(x) \neq 0$ whenever $x \neq 0$.

Let $\alpha$ be any family of subsets of $X$ satisfying the condition:

if $A, B \in \alpha$, then there exists a $C \in \alpha$ such that $A \cup B \subseteq C$.

For each $A \in \alpha$ and $\varepsilon>0$, let

$$
A_{\varepsilon}=\{(f, g) \in C(X) \times C(X):|f(x)-g(x)|<\varepsilon \text { for all } x \in A\} .
$$

Then it can be easily verified that the collection $\left\{A_{\varepsilon}: A \in \alpha, \varepsilon>0\right\}$ is a base for some uniformity on $C(X)$. We denote the space $C(X)$ with the topology induced by this uniformity be $C_{\alpha, u}(X)$. This topology is called the topology of uniform convergence on $\alpha$. For each $f \in C(X), A \in \alpha$ and $\varepsilon>0$, let

$$
<f, A, \varepsilon>=\{g \in C(X):|f(x)-g(x)|<\varepsilon \text { for all } x \in A\} .
$$

Then for each $f \in C(X)$, the collection $\{\langle f, A, \varepsilon\rangle: A \in \alpha, \varepsilon\rangle 0\}$ forms a neighborhood base at $f$ in $C_{\alpha, u}(X)$. Since the topology comes from a uniformity, then $C_{\alpha, u}(X)$ is completely regular. If $\alpha$ covers $X$, then $C_{\alpha, u}(X)$ is a Tychonoff space. When $\alpha=\{X\}$, we get the topology of uniform convergence, denoted by $C_{u}(X)$. It is clear that for any $\alpha, C_{\alpha, u}(X) \leq C_{u}(X)$. 
Now for each $A \in \alpha$, define the pseudo-seminorm $p_{A}$ on $C(X)$ by

$$
p_{A}(f)=\min \{1, \sup \{|f(x)|: x \in A\}\} .
$$

Also for each $A \in \alpha$ and $\varepsilon>0$, let

$$
V_{A, \varepsilon}=\left\{f \in C(X): p_{A}(f)<\varepsilon\right\} .
$$

Let

$$
\checkmark=\left\{V_{A, e}: A \in \alpha, \varepsilon>0\right\} .
$$

It can be easily shown that for each $f \in C(X), f+\checkmark \equiv\{f+V: V \in \checkmark\}$ forms a neighborhood base at $f$. We say that this topology is generated by the collection of pseudo-seminorms $\left\{p_{A}: A \in \alpha\right\}$. Note that if we choose $0<\varepsilon<1$, then for each $f \in C(X)$, we have

$$
f+V_{A, \varepsilon} \subseteq\langle f, A, \varepsilon>
$$

and

$$
<f, A, \frac{\varepsilon}{2}>\subseteq f+V_{A, \varepsilon}
$$

for all $A \in \alpha$. This shows that the topology of uniform convergence on $\alpha$ is the same as the topology generated by the collection of pseudo-seminorms $\left\{p_{A}: A \in \alpha\right\}$. We see from this point of view that $C_{\alpha, u}(X)$ is a topological group with respect to addition.

Observe that for $C^{*}(X)$ we can actually use the seminorms $p_{A}$ defined by

$$
p_{A}(f)=\sup \{|f(x)|: x \in A\} .
$$

Consequently, $C_{\alpha, u}^{*}(X)$ is a locally convex topological vector space. One might wonder when $C_{\alpha, u}(X)$ is a topological vector space. This is answered by the following theorem. In this theorem the term "bounded" refers to a subset of a space such that each restriction of a continuous realvalued function on the space to this subset is a bounded function.

THEOREM 1.1. The space $C_{\alpha, u}(X)$ is a linear topological space if and only if every element of $\alpha$ is bounded.

PROOF. If every element of $\alpha$ is bounded, then as in the case of $C_{\alpha, u}^{*}(X)$, we can actually use seminorms for $p_{A}$ when $A \in \alpha$.

For the converse, suppose that there is an $A \in \alpha$ which is not bounded. Then there is an $f \in C(X)$ and $\left\{x_{n}: n \in N\right\} \subseteq A$ such that $f\left(x_{n}\right) \geq n$ for each $n$. To show that scalar multiplication cannot be continuous in $C_{\alpha, u}(X)$, let $T$ be the scalar multiplication operator defined by $\left.T(t, g)\right)=t g$ for $t \in \Re$ and $g \in C_{\alpha, u}(X)$. We show that $T$ is not continuous at $(0, f)$. Let $V_{A, e}$ be a neighborhood of $f_{0}=T((0, f))$ in $C_{\alpha, u}(X)$, where $A \in \alpha$ and $0<\varepsilon<1$. Then for any neighborhood $U$ of 0 in $\Re$, there exists an $n \in N$ such that $\frac{1}{n} \in U$. But $\frac{1}{n} f\left(x_{n}\right) \geq 1>\varepsilon$, so that $T\left(\left(\frac{1}{n}, f\right)\right)=\frac{1}{n} f \notin V_{A, e^{*}}$

As a consequence of Theorem 1.1, if every element of $\alpha$ is pseudocompact (a space is pseudocompact if every continuous real-valued function on the space is bounded), then $C_{\alpha, u}(X)$ is a locally convex topological vector space. Conversely, if every element of $\alpha$ is $C$-embedded in $X$ (that is, every continuous real-valued function on the subspace has a continuous extension to $X$ ) and if $C_{\alpha, u}(X)$ is a topological vector space, then every element of $\alpha$ is pseudocompact.

For the rest of the paper, we are interested in the particular topology where $\alpha$ is the set of $\sigma$ compact (countable union of compact) subsets of $X$. In this case, we denote the space $C_{\alpha, u}(X)$ by $C_{\sigma, u}(X)$. This is called the topology of uniform convergence on $\sigma$-compact sets. Note that we get the 
same topology if we take the members of $\alpha$ to be the closures of the $\sigma$-compact subsets of $X$.

2. THE $\sigma$-COMPACT-OPEN TOPOLOGY.

For any subset $A$ of $X$ and any open subset $V$ of $\Re$, define

$$
[A, V]=\{f \in C(X): \overline{f(A)} \subseteq V\} .
$$

This agrees with the usual "set-open" terminology for the compact-open topology because $A$ is compact in this case. Now let $\sigma(X)$ be the set of $\sigma$-compact subsets of $X$, and let $\$$ be the set of bounded open intervals in $\Re$. For the $\sigma$-compact-open topology on $C(X)$, we take as subbase, the family

\section{$\{[A, B]: A \in \sigma(X), B \in \mathscr{B}\}$;}

and we denote this space by $C_{\sigma}(X)$. Note that the same topology is obtained by using $[\bar{A}, B]$, where $A \in \sigma(X)$ and $B \in \mathfrak{B}$. This is because for each $f \in C(X), f(\bar{A}) \subseteq \overline{f(A)}$; so that $\overline{f(\bar{A})}=\overline{f(A)}$. The fact that $C_{\sigma}(X)$ is a Tychonoff space can be proved in a manner similar to the proof of Lemma 5.1 in [9].

It is useful to relate basic open sets in $C_{\sigma}(X)$ to basic open sets in $C_{\sigma, u}(X)$, which is done in the following two lemmas.

LEMMA 2.1. Let $W=\cap_{i=1}^{n}\left[A_{i}, V_{\imath}\right]$ be a basic neighborhood of $f$ in $C_{\sigma}(X)$. Then there exists an $\varepsilon>0$ such that $\left\langle f, A_{1} \cup \cdots \cup A_{n}, \varepsilon>\subseteq W\right.$.

PROOF. For each $i$, since $\overline{f\left(A_{i}\right)}$ is compact, there is an $\varepsilon_{i}>0$ such that the $\varepsilon_{i}$-neighborhood of $\overline{f\left(A_{\mathfrak{i}}\right)}$ is contained in $V_{1}$. The conclusion now follows by taking $\varepsilon=\min \left\{\varepsilon_{1}, \ldots, \varepsilon_{n}\right\}$.

LEMMA 2.2. Let $A \in \sigma(X)$, and let $f \in C(X)$ be such that $f(A)$ is bounded. Then for each $\varepsilon>0$, there exists a basic open set $W$ in $C_{\sigma}(X)$ such that $f \in W \subseteq\langle f, A, \varepsilon\rangle$.

PROOF. Since $f(A)$ is bounded, there are open intervals $V_{1}, \ldots, V_{n}$ of length $\frac{\epsilon}{2}$ such that $\overline{f(A)} \subseteq \cup_{i=1}^{n} V_{i}$. For each $i$, let $A_{i}=A \cap f^{-1}\left(\bar{V}_{i}\right)$, and let $W_{i}$ be the $\frac{\varepsilon}{4}$ neighborhood of $V_{i}$ in $\Re$. Then each $A_{i} \in \sigma(X)$, and each $W_{3}$ is an open interval of length $\varepsilon$. So define $W=\cap_{i=1}^{n}\left[A_{2}, W_{1}\right]$, which is basic neighborhood of $f$ in $C_{\sigma}(X)$. It is straightforward to check that $W \subseteq\langle f, A, \varepsilon\rangle$.

Lemma 2.1 tells us that the $\sigma$-compact-open topology is coarser than or equal to the topology of uniform convergence on $\sigma$-compact sets. On the other hand, it is clear from definitions that the $\sigma$-compact-open topology is finer than or equal to the compact-open topology. Therefore we have the following general comparisons.

THEOREM 2.3. For every space $X, C_{k}(X) \leq C_{\sigma}(X) \leq C_{\sigma, u}(X) \leq C_{u}(X)$.

It is well-known that $C^{*}(X)$ is dense in $C_{k}(X)$. The same is true for ${ }^{\prime} C_{\sigma}(X)$, as we see in the next result.

THEOREM 2.4. For every space $X, C^{*}(X)$ is dense in $C_{\sigma}(X)$.

PROOF. If $\cap_{i=1}^{n}\left[A_{i}, V_{t}\right]$ is a basic open set in $C_{\sigma}(X)$ containing $f$, then $\cup_{i=1}^{n} V_{i}$ is contained in some interval $(a, b)$. Then if $g$ is defined by $g(x)=f(x)$ if $a \leq f(x) \leq b, g(x)=a$ if $f(x)<a$ and $g(x)=b$ if $b<f(x)$, we have that

$$
g \in C^{*}(X) \cap\left(\cap_{i=1}^{n}\left[A_{i}, V_{i}\right]\right)
$$

as desired.

Even though $C^{*}(X)$ is dense in $C_{\sigma}(X)$, the members of $C^{*}(X)$ play a special role in $C_{\sigma}(X)$ in the following sense. For each $f \in C(X)$, let $T_{f}: C_{\sigma}(X) \rightarrow C_{\sigma}(X)$ be the translation operator defined by $T_{f}(g)=f+g$ for all $g \in C_{\sigma}(X)$.

THEOREM 2.5. Let $f \in C(X)$. Then $T_{f}: C_{\sigma}(X) \rightarrow C_{\sigma}(X)$ is continuous if and only if $f \in C^{*}(X)$. 
PROOF. For the sufficiency, suppose that $f \in C^{*}(X)$ and that $[A, V]$ is a subbasic neighborhood of $T_{f}(g)$ in $C_{\sigma}(X)$ for some $g \in C_{\sigma}(X)$. Then by Lemma 2.1, there exists an $\varepsilon>0$ such that

$$
<T_{f}(g), A, \varepsilon>\subseteq[A, V] .
$$

Now $(f+g)(A)=T_{f}(g)(A)$ is bounded. Since $f \in C^{*}(X), g(A)=(f+g)(A)-f(A)$ is bounded. So by Lemma 2.2, there exists a basic open set $W$ in $C_{\sigma}(X)$ such that $g \in W \subseteq\langle g, A, \varepsilon\rangle$. We see that

$$
T_{f}(W) \subseteq T_{f}(<g, A, \varepsilon>) \subseteq<T_{f}(g), A, \varepsilon>\subseteq[A, V],
$$

and thus $T_{f}$ must be continuous at $g$.

For the necessity, suppose that $f \notin C^{*}(X)$. Then $f$ is unbounded on some $A \in \sigma(X)$. Now $[A,(-1,1)]$ is a neighborhood of $f_{0}=T_{f}(-f)$ in $C_{\sigma}(X)$. Suppose, by way of contradiction, that $T_{f}$ is continuous at $-f$. Then there would be a basic neighborhood $\cap_{i=1}^{n}\left[A_{i}, V_{i}\right]$ of $-f$ in $C_{\sigma}(X)$ such that

$$
T_{f}\left(\cap_{i=1}^{n}\left[A_{i}, V_{v}\right]\right) \subseteq[A,(-1,1)] .
$$

Since $-f$ would be bounded on $\cup_{i=1}^{n} A_{i}$, so would $f$. Therefore there would exist some $x \in A \backslash \overline{U_{i=1}^{n} A_{i}}$. Then let $\phi \in C(X)$ be such that $\phi(x)=1$ and $\phi(y)=0$ for all $y \in \overline{U_{i=1}^{n} A_{z}}$, and define $h=\phi-f$. Clearly $h \in \cap_{i=1}^{n}\left[A_{\imath}, V_{i}\right]$, so that $f+h=T_{f}(h) \in[A,(-1,1)]$. But then $1=(f+h)(x) \in(-1,1)$, which is a contradiction. Therefore $T_{f}$ is not continuous at $-f$.

COROLLARY 2.6. The following are equivalent.

(1) $C_{\sigma}(X)$ is a topological vector space.

(2) $C_{\sigma}(X)$ is a topological semigroup under addition.

(3) $C_{\sigma}(X)=C_{\sigma}^{*}(X)$.

(4) $X$ is pseudocompact.

When studying function spaces, it is sometimes useful to use induced functions. That is, if $\phi: X \rightarrow Y$ is a continuous function, define $\phi^{*}: C(Y) \rightarrow C(X)$ by $\phi^{*}(g)=g \circ \phi$ for all $g \in C(Y)$. We can establish the following theorem much like the corresponding theorem for the compact-open topology (cf. [10]). In this theorem, the definition of $\phi: X \rightarrow Y$ being a $\sigma$-compact-covering map is that each $\sigma$ compact subset of $Y$ is contained in the image of some $\sigma$-compact subset of $X$ under $\phi$.

THEOREM 2.7. If $\phi: X \rightarrow Y$ is continuous, then $\phi^{*}: C_{\sigma}(Y) \rightarrow C_{\sigma}(X)$ is continuous. Furthermore, if $\phi$ is a $\sigma$-compact-covering map, then $\phi^{*}$ is an embedding.

3. COMPARISON OF TOPOLOGIES.

We have already seen that

$$
C_{k}(X) \leq C_{\sigma}(X) \leq C_{\sigma, u}(X) \leq C_{u}(X) .
$$

In this section we determine when then inequalities are equalities and give examples to illustrate the differences.

THEOREM 3.1. For every space $X, C_{k}(X)=C_{\sigma}(X)$ if and only if the closure of each $\sigma$-compact subset of $X$ is compact.

PROOF. The sufficiency is straightforward. For the necessity, suppose that $C_{k}(X)=C_{\sigma}(X)$ and let $A \in \sigma(X)$. Then there exist a compact set $K$ in $X$ and an open set $V$ in $\Re$ such that

$$
f_{0} \in[K, V] \subseteq[A, \Re \backslash\{1\}] \text {. }
$$

Suppose, by way of contradiction, that there exists an $x \in \bar{A} \backslash K$. Then there would be some $f \in C(X)$ such that $f(x)=1$ and $f(y)=0$ for all $y \in K$. But then

$$
f \in[K, V] \backslash[A, \Re \backslash\{1\}],
$$

which is a contradiction. Therefore $\bar{A} \subseteq K$, so that $\bar{A}$ is compact. 
COROLLARY 3.2. If $C_{k}(X)=C_{\sigma}(X)$, then $X$ is countably compact.

THEOREM 3.3. For every space $X, C_{\sigma}(X)=C_{\sigma, u}(X)$ if and only if $X$ is pseudocompact.

PROOF. If $X$ is pseudocompact, then for each $A \in \sigma(X)$ and $f \in C(X), f(A)$ is bounded. Therefore by Lemma 2.2 , we have that $C_{\sigma}(X)=C_{\sigma, u}(X)$.

For the converse, suppose that $X$ is not pseudocompact. Then there is some $f \in C(X)$ and $A \in \sigma(X)$ such that $f(A)$ is unbounded. Now for any $\varepsilon>0,\langle f, A, \varepsilon\rangle$ cannot be a neighborhood of $f$ in $C_{\sigma}(X)$. This is because for arbitrary open $W$ in $C_{\sigma}(X)$ there is some $g \in W \cap C^{*}(X)$ by Theorem 2.4; but $g \notin<f, A, \varepsilon>$. It follows that $C_{\sigma}(X)<C_{\sigma, u}(X)$.

COROLLARY 3.4. If every $\sigma$-compact subset of $X$ has compact closure, then $C_{k}(X)=C_{\sigma, u}(X)$.

THEOREM 3.5. For every space $X, C_{\sigma, u}(X)=C_{u}(X)$ if and only if $X$ contains a dense $\sigma$ compact subset.

PROOF. Suppose that $X$ contains an $A \in \sigma(X)$ which is dense. Then for each $f \in C(X)$ and $\varepsilon>0$,

$$
<f, A, \frac{\varepsilon}{2}>\subseteq<f, X, \varepsilon>.
$$

Thus $\langle f, X, \varepsilon\rangle$ is a neighborhood of $f$ in $C_{\sigma, u}(X)$, so that $C_{\sigma, u}(X)=C_{u}(X)$.

Conversely, let $C_{\sigma, u}(X)=C_{u}(X)$, and consider $\left\langle f_{0}, X, \varepsilon>\right.$ where $0<\varepsilon<1$. Then there exists an $A \in \sigma(X)$ and $\delta>0$ such that

$$
\left\langle f_{0}, A, \delta>\subseteq\left\langle f_{0}, X, \varepsilon>,\right.\right.
$$

Suppose, by way of contradiction, that there is an $x \in X \backslash \bar{A}$. Then there would be some $f \in C(X)$ with $f(x)=1$ and $f(y)=0$ for all $y \in \bar{A}$. But this would mean that

$$
f \in\left\langle f_{0}, A, \delta>\backslash<f_{0}, X, \varepsilon\right\rangle
$$

which is a contradiction. It follows that $X=\bar{A}$.

COROLLARY 3.6. If $X$ is separable, then $C_{\sigma, u}(X)=C_{u}(X)$.

We end this section by looking at some examples which illustrate all possible inequalities between these function spaces.

EXAMPLE 3.7. If $X$ is any compact space, then

$$
C_{k}(X)=C_{\sigma}(X)=C_{\sigma, u}(X)=C_{u}(X) .
$$

EXAMPLE 3.8. If $X$ is an uncountable discrete space, then

$$
C_{k}(X)<C_{\sigma}(X)<C_{\sigma, u}(X)<C_{u}(X) .
$$

EXAMPLE 3.9. If $X=N$ or $X=\Re$, then

$$
C_{k}(X)<C_{\sigma}(X)<C_{\sigma, u}(X)=C_{u}(X) .
$$

EXAMPLE 3.10. Let $X$ be the space of countable ordinals with the order topology. Then each $\sigma$-compact subset of $X$ has compact closure, but $X$ does not contain a dense $\sigma$-compact subset. Therefore

$$
C_{k}(X)=C_{\sigma}(X)=C_{\sigma, u}(X)<C_{u}(X) .
$$

EXAMPLE 3.11. Let $\mathbf{N}^{*}=\beta N \mid N$, where $\beta N$ is the Stone-Čech compactification of $N$. Let $p$ be a point of $\mathrm{N}^{*}$ which is not a $P$-point (cf. [11]). Define $X$ to be $\mathrm{N}^{*} \backslash\{p\}$. Then $X$ is countably compact, but does not have a dense $\sigma$-compact subset (since points are not $G_{\delta}$-sets). Also since $p$ is not a $P$-point, there is some $\sigma$-compact subset of $X$ which has non-compact closure. Therefore

$$
C_{k}(X)<C_{\sigma}(X)=C_{\sigma, u}(X)<C_{u}(X) \text {. }
$$


EXAMPLE 3.12. Let $X=\beta N \backslash\{p\}$, where $p \in N^{*}$ (see Example 3.11). Then $X$ is countably compact and separable, but not compact. Therefore

$$
C_{k}(X)<C_{\sigma}(X)=C_{\sigma, u}(X)=C_{u}(X) .
$$

Note that this shows that the converse of Corollary 3.2 is not true. An example of a space which is not countably compact but which has these same function space relations is the space $\Psi$ in $5 \mathrm{I}$ of [4].

4. ADDITIONAL PROPERTIES.

Theorem 3.5 can be expanded to include some additional properties of the spaces $C_{\sigma}(X)$ and $C_{\sigma, u}(X)$.

THEOREM 4.1. The following are equivalent.

(1) $C_{\sigma, u}(X)$ is (completely) metrizable.

(2) $C_{\sigma, u}(X)$ is first countable.

(3) $\left\{f_{0}\right\}$ is a $G_{\delta}$-set in $C_{\sigma, u}(X)$.

(4) $C_{\sigma}(X)$ is submetrizable.

(5) $C_{\sigma}(X)$ is first countable.

(6) $\left\{f_{0}\right\}$ is a $G_{\delta}$-set in $C_{\sigma}(X)$.

(7) $X$ contains a dense $\sigma$-compact subset.

PROOF. That (7) implies (1) follows from Theorem 3.5 since $C_{u}(X)$ is always completely metrizable.

To prove that (3) implies (7), suppose that $X$ does not contain a dense $\sigma$-compact subset. Now a $G_{\delta^{-}}$-set is a subset which can be written as a countable intersection of open subsets. So for each $n \in N$, let $\left\langle f_{0}, A_{n}, \varepsilon_{n}>\right.$ be any basic neighborhood of $f_{0}$ in $C_{\sigma, u}(X)$. We know that there is some $x \in X \backslash \overline{\cup_{n=1}^{\infty} A_{n}}$. Let $f \in C(X)$ be such that $f(x)=1$ and $f(y)=0$ for all $y \in \overline{U_{n=1}^{\infty} A_{n}}$. Then

$$
f \in \cap_{n=1}^{\infty}<f_{0}, A_{n}, \varepsilon_{n}>.
$$

But since $f \neq f_{0}$, we see that $\left\{f_{0}\right\}$ is not a $G_{\sigma}$-set in $C_{\sigma, u}(X)$.

Since (6) implies (3), it remains only to show that (7) implies (4) and (5). Suppose that $X$ contains a dense $\sigma$-compact subset $D$. Let $f$ be any element of $C_{\sigma}(X)$. We need to demonstrate that $f$ has a countable base in $C_{\sigma}(X)$. We may take for $\mathbb{B}$ the family of bounded open intervals in $\Re$ with rational endpoints. Also let $e$ be the closures of the members of 9 . Then define

$$
\mathcal{l}=\left\{f^{-1}(C) \cap D: C \in \mathrm{e}\right\} .
$$

Since $D$ is $\sigma$-compact, $\boldsymbol{l}$ is a countable subfamily of $\sigma(X)$. Since $\Phi_{B}$ is also countable, it now suffices to show that for each subbasic neighborhood $[S, V]$ of $f$ in $C_{\sigma}(X)$, there is an $A \in \mathcal{U}$ and $B \in \Phi$ such that $f \in[A, B] \subseteq[S, V]$.

If $f \in[S, V]$, then $\overline{f(S)} \subseteq V$. Hence since $\overline{f(S)}$ is compact, there exist $C \in \mathrm{C}$ and $B \in \mathscr{B}$ such that

$$
\overline{f(S)} \subseteq \text { int } C \subseteq C \subseteq B \subseteq V .
$$

Therefore if we define $A=f^{-1}(C) \cap D$, then we have

$$
\overline{f(A)} \subseteq \overline{f\left(f^{-1}(C)\right) \cap f(D)} \subseteq C \cap \overline{f(D)} \subseteq B .
$$

This means that $f \in[A, B]$. To show that $[A, B] \subseteq[S, V]$, let $g \in[A, B]$ and let $t \in \overline{g(S)}$. If $W$ is a neighborhood of $t$, then $g^{-1}(W) \cap S \neq 0$. Since

$$
S \subseteq f^{-1} \overline{(f(S))} \subseteq f^{-1}(\text { int } C),
$$


then $g^{-1}(W) \cap f^{-1}($ int $C) \neq 0$. But then

$$
g^{-1}(W) \cap f^{-1}(\text { int } C) \cap D \neq 0 .
$$

so that $g^{-1}(W) \cap A \neq \emptyset$. Therefore $W \cap g(A) \neq \emptyset$, and thus $t \in \overline{g(A)} \subseteq B$. It follows that $g \in[S, V]$, so that $f \in[A, B] \subseteq[S, V]$. We can now conclude that $f$ has a countable base in $C_{\sigma}(X)$.

Finally, to show that (7) implies (4), write the dense set $D=\cup_{n=1}^{\infty} A_{n}$, where each $A_{n}$ is a compact subset of $X$. A submetrizable space is one which can be mapped into a metric space by a continuous injection. (Note that singleton sets are $G_{\delta}$-sets in a submetrizable space.) Define $Y$ to be the disjoint topological sum $\sum_{n=1}^{\infty} A_{n}$, and let $\phi: Y \rightarrow X$ be the natural map. Then the induced function $\phi^{*}: C_{\sigma}(X) \rightarrow C_{\sigma}(Y)$ is continuous by Theorem 2.7. Since $\phi(Y)$ is dense in $X, \phi^{*}$ is an injection. Now the sum map

$$
S: C_{\sigma}\left(\sum_{n=1}^{\infty} A_{n}\right) \rightarrow \prod_{n=1}^{\infty} C_{\sigma}\left(A_{n}\right)=\prod_{n=1}^{\infty} C_{k}\left(A_{n}\right)
$$

is a continuous bijection, since it is a homeomorphism when defined on $C_{k}\left(\sum_{n=1}^{\infty} A_{n}\right.$ ) (cf. [6]). Therefore $S \circ \phi^{*}$ is a continuous injection from $C_{\sigma}(X)$ into the metrizable space $\prod_{n=1}^{\infty} C_{k}\left(A_{n}\right)$, which makes $C_{\sigma}(X)$ submetrizable.

For our last topic, we consider the separability of $C_{\sigma}(X)$, and the weaker property of having the countable chain condition (i.e., every pairwise disjoint family of nonempty open subsets is countable). We see from the following theorem and Theorem 3.3 that $C_{\sigma, u}(X)$ is separable (has ccc) if and only if $C_{\sigma}(X)$ is separable (has ccc).

THEOREM 4.2. If $C_{\sigma}(X)$ has the countable chain condition, then $X$ is pseudocompact.

PROOF. Suppose that $X$ is not pseudocompact. Then there is a closed $C$-embedding $\phi: N \rightarrow X$. So by Theorem 2.7, the induced function $\phi^{*}: C_{\sigma}(X) \rightarrow C_{\sigma}(\mathrm{N})$ is continuous. Since the embedding is a $C$-embedding, $\phi^{*}$ is a surjection.

We show that $C_{\sigma}(\mathrm{N})$ does not have the countable chain condition. Then since $\phi^{*}$ is a continuous surjection, $C_{\sigma}(X)$ will also not have the countable chain condition. Let $U=(0,1)$ and $V=(1,2)$, and for each $A$ in the power set $\Phi(N)$ of $N$, define

$$
S_{A}=[A, U] \cap[N \backslash A, V] .
$$

Then $S_{A}$ is a nonempty basic open set in $C_{\sigma}(\mathrm{N})$. Suppose that $A, B \in \Phi(\mathbb{N})$ with $A \neq B$. Without loss of generality, say there is some $x \in A \backslash B$. If $f \in S_{A}$, then $f(x) \in U$; but if $f \in S_{B}$, then $f(x) \in V$. Since $U \cap V=\emptyset, S_{A} \cap S_{B}=\emptyset$. Therefore $\left\{S_{A}: A \in \mathscr{P}(\mathrm{N})\right\}$ is an uncountable pairwise disjoint family of nonempty open subsets of $C_{\sigma}(\mathbf{N})$.

To complete the characterization of $C_{\sigma}(X)$ being separable, we need the following lemma. It is a consequence of Theorem 8.17 in [4]; however, we give a simple direct proof.

LEMMA 4.3. Every pseudocompact submetrizable (Tychonoff) space is compact and metrizable.

PROOF. Suppose topological space $(X, \tau)$ is pseudocompact and has a metric $d$ which generates a strictly coarser topology $\tau_{d}$ on $X$. Then there exists an $x \in U \in \tau \backslash \tau_{d}$ and sequence $\left(x_{n}\right)$ of distinct points of $X \backslash U$ such that $\left(x_{n}\right)$ converges to $x$ in $\tau_{d}$. For each $n$, let $B_{n}$ be an open neighborhood of $x_{n}$ in $\tau_{d}$ having diameter less than $\frac{1}{n}$ and such that $B_{i} \cap B_{j}=0$ for $i \neq j$. Now let $V \in \tau$ be a neighborhood of $x$ whose closure, $\bar{V}$, in $\tau$ is contained in $U$. For each $n$, define $V_{n}=B_{n} \mid \bar{V}$, which is a neighborhood of $x_{n}$ in $\tau$. Note that $\left\{V_{n}: n \in N\right\}$ is a discrete family in $r$. For each $n$, choose $f_{n} \in C(X)$ such that $f_{n}\left(x_{n}\right)=n$ and $f(y)=0$ for all $y \in X \backslash V_{n}$. Define $f \in C(X)$ by $f(y)=f_{n}(y)$ if $y \in V_{n}$ and $f(y)=0$ otherwise. Since $f$ is unbounded, this contradicts $(X, \tau)$ being pseudocompact.

THEOREM 4.4. The space $C_{\sigma}(X)$ is separable if and only if $X$ is compact and metrizable. 
PROOF. If $C_{\sigma}(X)$ is separable, then the smaller space $C_{k}(X)$ is separable. This means that $X$ is submetrizable. The result now follows from Theorem 4.2 and Lemma 4.3.

QUESTION 4.5. When is $C_{\sigma}(X)$ metrizable? In particular, is $C_{\sigma}(\mathrm{N})$ metrizable or even normal?

ACKNOWLEDGEMENT. The first author's research is supported by the Council of Scientific and Industrial Research, India.

\section{REFERENCES}

1. ARENS, R., A topology for spaces of transformations, Annals of Math. 47 (1946), 480-495.

2. ARENS, R. \& DUGUNDJI, J., Topologies for function spaces, Pacific J. Math. 1 (1951), 5-31.

3. FOX, R.H., On topologies for function spaces, Bull. Amer. Math. Soc. 51 (1945), 429-432.

4. GILLMAN, L. \& JERISON, M., Rings of continuous functions, D. Van Nostrand, Princeton, New Jersey, 1960.

5. GULICK, D., The $\sigma$-compact-open topology and its relatives, Math. Scand. 30 (1972), 159-176.

6. JACKSON, J.R., Comparison of topologies on function spaces, Proc. Amer. Math. Soc. 3 (1952), 156-158.

7. KUNDU, S., Spaces of continuous linear functionals: something old and something new, Topology Proc. 14 (1989), 113-129.

8. KUNDU, S., McCOY, R.A., \& OKUYAMA, A., Spaces of continuous linear functionals on $C_{k}(X)$, Mathematica Japonica 34 (1989), 775-787.

9. McCOY, R.A. \& NTANTU, I., Completeness properties of function spaces, Topology and Appl. 22 (1986), 191-206.

10. McCOY, R.A. \& NTANTU, I., Topological properties of spaces of continuous functions, Lecture Notes in Mathematics, $\underline{1315}$, Springer-Verlag, Berlin, 1988.

11. VAN MILL, J., An introduction to $\beta \omega$, Handbook of Set-theoretic Topology, North-Holland, Amsterdam (1984) 503-567. 


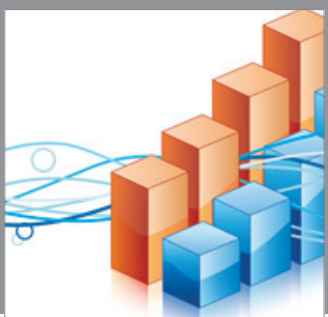

Advances in

Operations Research

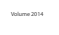

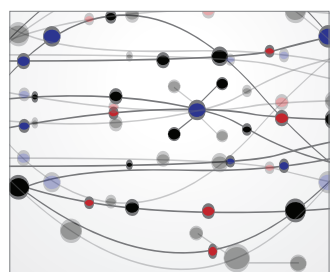

\section{The Scientific} World Journal
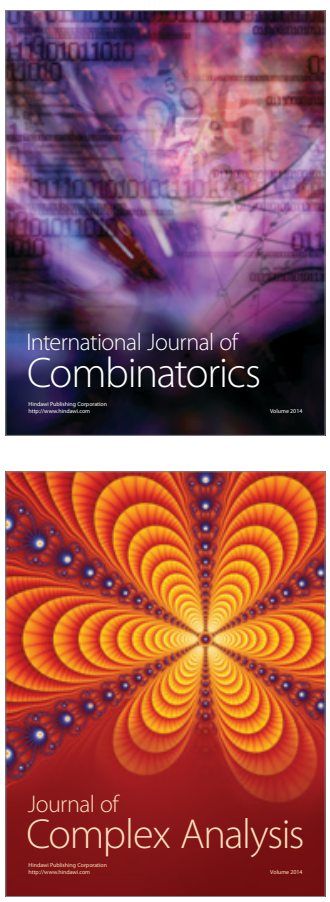

International Journal of

Mathematics and

Mathematical

Sciences
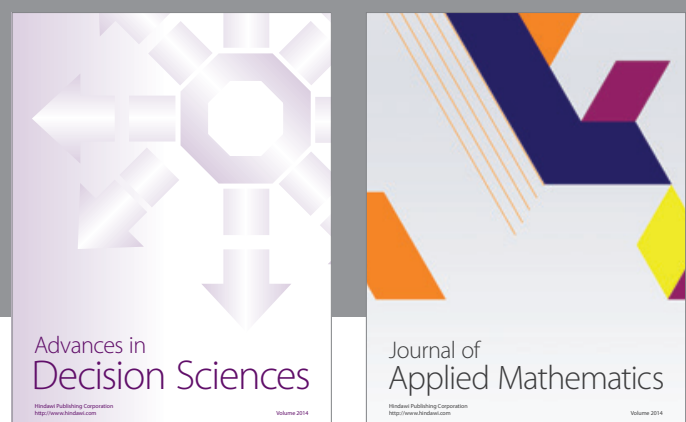

Journal of

Applied Mathematics
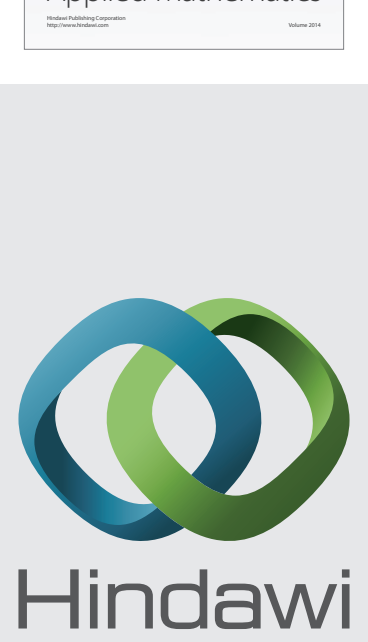

Submit your manuscripts at http://www.hindawi.com
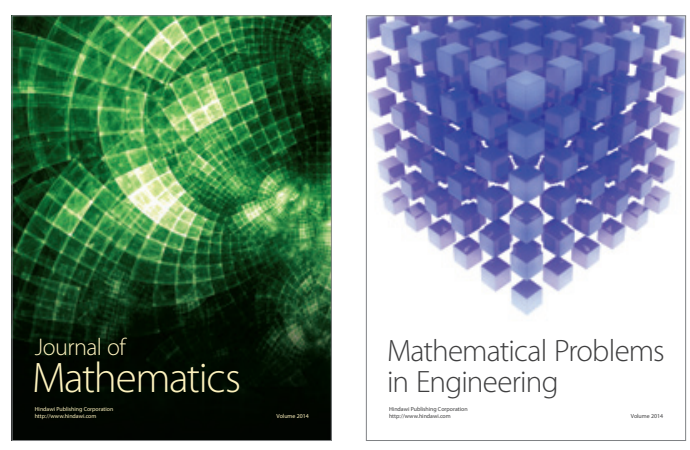

Mathematical Problems in Engineering
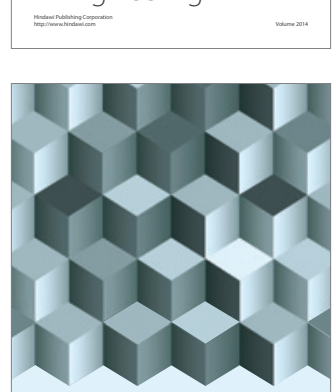

Journal of

Function Spaces
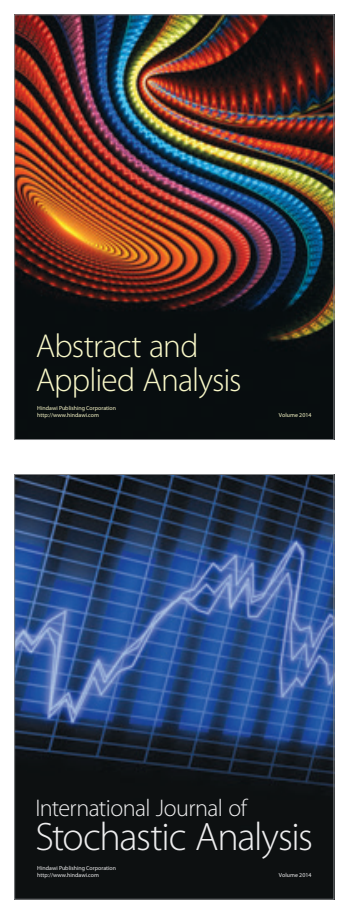

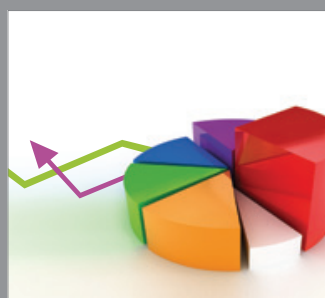

ournal of

Probability and Statistics

Promensencen
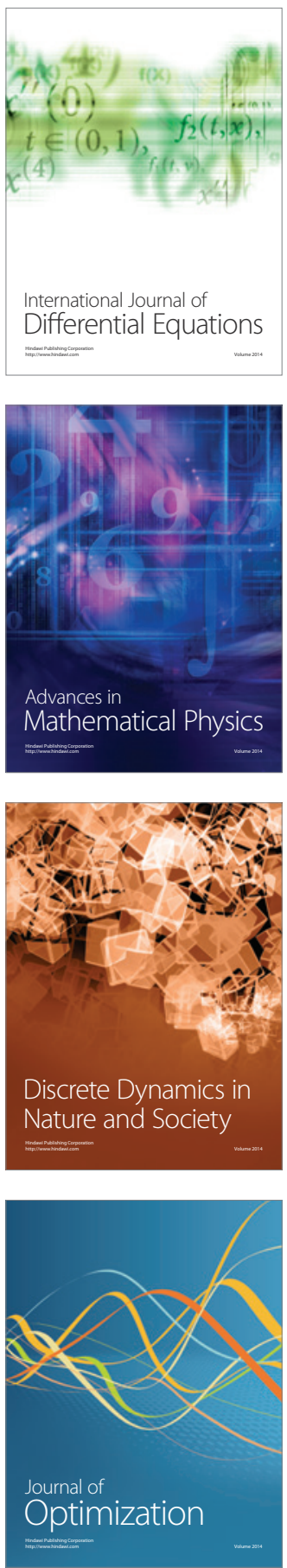